\title{
A Combined Method for Texture Analysis and Its Application
}

\author{
Yongping Zhang ${ }^{1}$ and Ruili Wang ${ }^{2}$ \\ ${ }^{1}$ Bioengineering Institute, The University of Auckland \\ Level 6, 70 Symonds St., Auckland, New Zealand \\ Zhangyp1963 ayahoo.com \\ ${ }^{2}$ Institute of Information Sciences and Technology, Massey University \\ Private Bag 11 222, Palmerston North, New Zealand \\ R.Wang@Massey.ac.nz
}

\begin{abstract}
In this paper, a rotational invariant feature set is introduced for texture classification, based on wavelet transformation in combination with cooccurrence probabilities. Using this combined method, through wavelet decomposition and reconstruction, an approximation image and a new details image are generated. Beside of using the statistic approximation and the new details respectively, the joint distribution of the original and the new detail image is computed, and seven novel digital features are derived from the joint probability. By combination with a MLP neural network, our method has successfully applied to pollen discrimination. In experiments with sixteen types of airborne pollen grains, more than 95 percent pollen images are correctly classified.
\end{abstract}

\section{Introduction}

Texture feature is one of the most widely used visual features in pattern recognition and computer vision. Texture contains important information about the surface structure of objects and their relationship to the surrounding environment [1].

Many studies have shown that use of wavelet transforms for texture description can achieve good classification performance [2-10]. Smith and Chang used the statistic feature of subbands as the texture representation [4]. Gross et al. characterised texture by using wavelet transform in combination with KL expansion [5].

A combined approach of wavelet transform with co-occurrence matrix was also carried out by Thyagarajan et al. in [6]. In this paper, a novel co-occurrence matrix is introduced for texture description based on wavelet transforms, such matrix is corresponding to the joint distribution of the original greyscale image and the details derived from wavelet transforms.

Distinguishing the pollen species through the analysis of their surface images has become a new application field of computer vision [11-16].

In the present work, we use our combined algorithm to extract texture features of surface images of pollen grains and use a multilayer perceptron (MLP) neural network to classify the extracted feature vectors. 


\section{Wavlet-Based Feature Set}

In this section, we introduce the combined features. First we define the details reconstruction and then we introduce a novel method for feature extraction.

On the each level of orthogonal wavelet decomposition, one approximation image and three details images can be got; those details images are horizontal, vertical and diagonal details respectively. For image analysis, the four subband images are usually assumed to be independent. In the present study, we reconstruct a new details image using the three details subbands, and compute the corresponding statistic features of the approximation sunband and the new details image, and to compute the joint distribution of the original image and the new details image. The scheme for feature extraction as shown in Fig. 1.

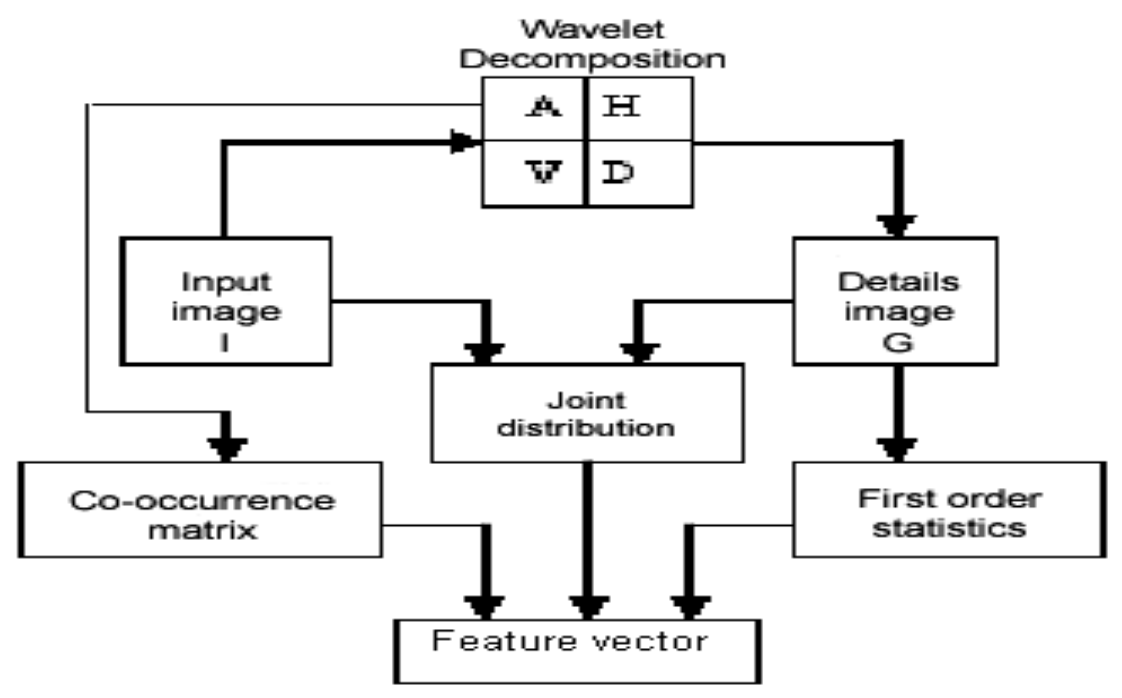

Fig. 1. The scheme of texture feature extraction based on wavelet decomposition

To form effective feature vector, we compute the joint distribution of the original image and its detail image. First of all, like the gray level co_occurrence matrix, a cooccurrence matrix $\mathrm{Q}$ is calculated as:

$$
q(i, j)=\frac{\#\{p \in I \mid I(p)=i, G(p)=j\}}{\# I}
$$

where $G$ presents the details image reconstructed from the wavelet decomposition of the input image $I$, which is re-quantized (discretized) to certain grey levels.

Based on this co-occurrence matrix $\mathrm{Q}$, we compute the following seven features: small detail emphasis (SDE), large detail emphasis (LGE), gray distribution nonuniformity (GDNU), details distribution non-uniformity (DDNU), energy (ENE), Entropy (ENT) and inverse difference moment (IDM). We can call the above seven features as grey-detail co-occurrence matrix (GDCM) features. 


\section{Pollen Image Classification}

We have applied the new texture features to discriminate the airborne pollen grains. The classification results verify the robustness of our combined method by providing high percentage of correct classification for texture images obtained from sixteen types of pollen grains.

In this research, sixteen types of airborne pollen grains are considered and their typical surface images shown in Fig. 2.

We computed 15 features for each sample using our method. For feature selection, the 15 features were extracted from 15 samples of each type of pollen grains, and the ratio of interclass/intraclass distance was used. The resultant 7 features: GLCM features ENT and IDM, GDCM features GDNU, ENE and IDM, detail features mean and standard deviation are selected to represent pollen texture.

For classification of pollen images, the MLP neural network of $7 \times 15 \times 16$ was employed, and back-propagation algorithm was used for network training. In this experiment, 95.4 percent of pollen images are correctly classified.
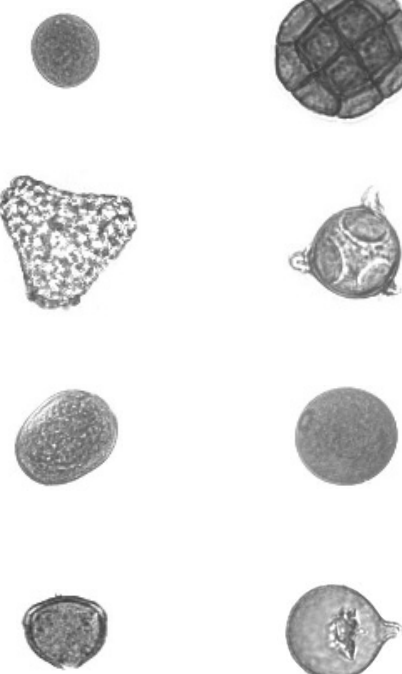
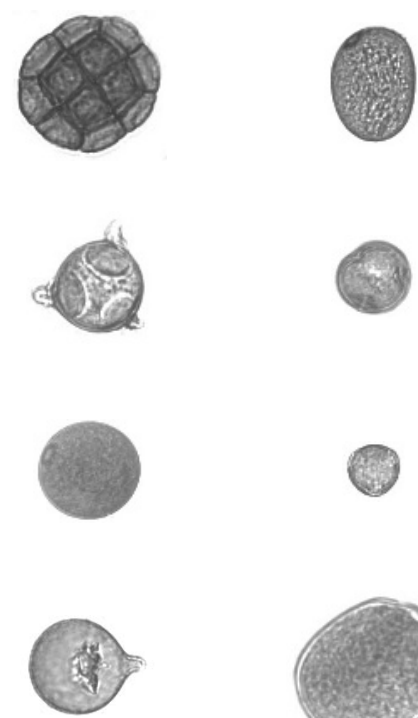
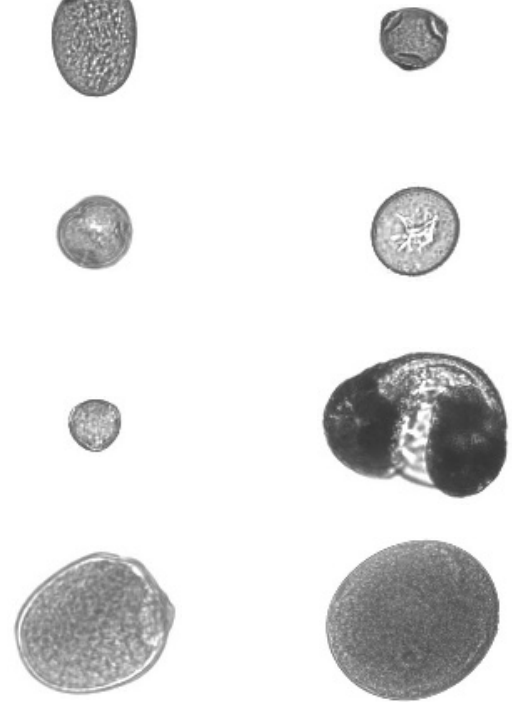

Fig. 2. The pollen types analysed in this research. From left to right and top to bottom: AC (Agrostis capillaris), $\mathrm{AD}$ (Acacia dealbata), $\mathrm{AP}($ Alopecuris pratensis $), \mathrm{BP}($ Betula pendula $)$, $\mathrm{CD}($ Cyathea dealbata $), \mathrm{CL}($ Corylus $), \mathrm{CR}($ Coprosma robusta $), \mathrm{CT}($ Cedar tree $), \mathrm{FA}($ Festuca arundibaceae), $\mathrm{PM}($ Phalaris minor), $\mathrm{PO}$ (Platanus orientalis), $\mathrm{PR}$ (Pinus radiata), QR (Quercus robur), SG(Sequiadendron gigantea), TA(Triticum aestivum), ZM(Zea mays) 


\section{Conclusions}

A novel combined feature set has been developed and evaluated for texture classification.

The proposed method also has been successfully applied to identification of pollen grains. By feature selection, seven features are used as texture descriptors to present pollen images, and the MLP neural network is used to discriminate features of sixteen type pollen grains. A classification rate of more than 95 percent is achieved.

\section{References}

1. Ojala, T., Pietikainen, M.: A comparative study of texture measures with classification based on feature distributions, Pattern Recognition, Vol. 29(1), (1996) 51-59.

2. Randen, T., Hus Øy, H.J.: Filtering for texture classification: A comparative study, IEEE Transactions on Pattern Analysis and Machine Intelligence, Vol. 21(4), (1999) 291-310.

3. Reed, T.R., Buf, J.M.H.: A review of recent texture segmentation and feature extraction techniques", Computer Vision, Image Processing and Graphics, Vol. 57(3) (1993) 359-372.

4. Smith, J.R., Chang, S.: Transform features for texture classification and discrimination in large image databases, In Proc. IEEE Int. Conf. on Image Proc. (1994).

5. Gross, M. H., Koch, R., Lippert, L., Dreger. A.: "Multiscale image texture analysis in wavelet spaces, In Proc. IEEE Int. Conf. on Image Proc. (1994).

6. Thyagarajan, K.S., Nguyen, T., Persons, C.: A maximum likelihood approach to texture classification using wavelet transform, In Proc. IEEE Int. Conf. on Image Proc.(1994).

7. Do, M.N., Vetterli, M.: Texture similarity measurement using Kullback-Leibler distance on wavelet subbands, In Proc. of IEEE Int. Conf. on Image Proc. (2000).

8. Ma, W.Y., Manjunath, B.S.: A texture thesaurus for browsing large aerial photographs, Journal of the American Society for Information Science Vol. 49(7), (1998) 633-648.

9. Manjunath, B.S., Ma, W.Y.: Texture features for browsing and retrieval of large image data, IEEE Transactions on Pattern Analysis and Machine Intelligence, Vol. 18, (1996) 837-842.

10. Charalampidis, D., Kasparis, T.: Wavelet-based rotational invariant roughness features for texture classification and segmentation, IEEE Trans. on Image Proc., Vol. 11(8), (2002) 825-837.

11. Stillman, E.C., Flenley, J.R.: The needs and prospects for automation in palynology, Quaternary Science Reviews Vol. 15 (1996) 15.

12. Fountain, D.W.: Pollen and inhalant allergy, Biologist Vol.49 (1), (2002) 5-9.

13. Trelor, W.J.: Digital image processing techniques and their application to the automation of palynology, Ph. D. Thesis (1992), University of Hull, Hull UK.

14. Li, P., Flenley J.R.: Pollen texture identification using neural networks, Grana Vol. 38 (1999) 59-64

15. Langford, M., Taylor, G.E., Flenley, J.R.: Computerised identification of pollen grains by texture analysis, Review of Palaeobotany and Palynology, Vol. 64, (1990) 197-203

16. Ronneberger, O.: Automated pollen recognition using grey scale invariants on $3 \mathrm{D}$ volume image data. $2^{\text {nd }}$ European Symposium on Aerobiology. (2000) p.3. Vienna, Austria.

17. Mallat, S.: A Wavelet Tour of Signal Processing, Academic Press (1998), San Diego

18. Pandya, A.S., Macy, R.B.: Pattern Recognition with Neural Networks in C++. CRC and IEEE Press, (1996) Florida. 